\title{
Evaluación de la efectividad de una composición etnofarmacológica dirigida a la cura y/o alivio de la gastritis
}

\section{Evaluation of the effectiveness of a composition etnopharmacologic directed to the cure and/or lightening of the gastritis}

\author{
Carlos Ariel Rentería Jiménez ${ }^{1}$, Manuel Arcindo García Martínez ${ }^{2}$
}

\section{RESUMEN}

Este estudio tuvo como objetivo evaluar la efectividad antigástrica de una composición etnofarmacológica preparada a partir de cuatro especies de plantas medicinales: caléndula (Calendula oficinales), llantén (Plantago major), botoncillo (Spilanthes americana) y micay (Axonopus micay), para lo que se administraron dosis diarias de esta composición a pacientes declarados portadores de gastritis por parte de un médico facultativo, haciéndoles seguimiento mediante expediente clínico, a fin de sistematizar y documentar el procedimiento médico tradicional, en términos de sus componentes, eficacia y dosificación. Se observó que la composición etnofarmacéutica alivia la gastritis, la efectividad que se obtuvo fue bastante significativa en relación con el tratamiento y las personas mostraron porcentajes de recuperación y mejoría sintomática, lo que se evidencia principalmente en los diferentes testimonios de los pacientes sometidos al tratamiento. Los análisis fitoquímicos realizados a la composición etnofarmacológica, mostraron resultados de presunción positiva para la presencia de compuestos fenólicos, cumarinas y leucoantocianidinas, compuestos de reconocida acción antigástrica.

Palabras clave: Acción antigástrica; Composición etnofarmacológica; Gastritis; Plantas medicinales.

\section{ABSTRACT}

The present study had as objective to evaluate the antigastric effectiveness of a prepared etnofarmacológica composition from four (4) species of medicinal plants: caléndula (Calendula oficinales), llantén (Plantago major), botoncillo (Spilanthes americana) and micay (Axonopus micay). For which, they administered daily doses of this etnofarmacologica composition to declared patients carrying of gastritis on the part of a facultative doctor, realising pursuit to them by means of clinical file, in order to systematize and to document the traditional medical procedure, in terms of its components, effectiveness and dosage. It was observed that the etnofharmaceutic composition alleviates the gastritis, the effectiveness that was obtained was quite significant in relation to the treatment and the people showed to percentage of recovery and symptomatic improvement related to the gastritis, which is demonstrated mainly in the different testimonies of the put under patients from the etnofhamacologic treatment. The realised fitoquimicos analyses to the etnofarmacológica composition, showed results of positive presumption for the phenolic compound presence, cumarinas and leucoantocianidinas composed of recognized antigastric action.

Keywords: Antigastric action; Etnopharmacologic composition; Medicinal plants; Gastritis.
1. Licenciado en Biologíay Química, Magister en Docencia de la Química. Investigador de Proyectos Especiales, Instituto de Investigaciones Ambientales del Pacífico (IIAP), Quibdó, Colombia.

e-mail:crenteria@iiap.org.co

2. Investigador Médico Tradicional. Contratista IIAP, Quibdó, Colombia. e-mail:crenteria@iiap.org.co Recibido: 15 de marzo de 2011 Aceptado: 19 de abril de 2011 


\section{INTRODUCCIÓN}

Respecto a la situación de salud en Colombia y específicamente en el Pacífico colombiano, se debe indicar que una de las características de estas sociedades es que los índices de salud son alarmantes. A esto se suma la falta de políticas apropiadas y la falta de medicamentos económicos accesibles a las mayorías y con credibilidad para los pobladores, lo que ocasiona que poblaciones de áreas rurales no dispongan de medicamentos esenciales para atender sus problemas de salud. Esta situación, sin duda podría mejorar si los recursos naturales que son tan abundantes en nuestra región, se aprovecharan para la extracción de fármacos que se puedan emplear en la elaboración de medicamentos de bajo costo.

Diversos estudios adelantados por el Instituto de Investigaciones Ambientales del Pacífico (IIAP), demuestran cómo esta información popular y tradicional sobre el uso de plantas medicinales es un acervo cultural celosamente guardado por las generaciones a través del tiempo. El uso de plantas medicinales constituye una tradición ancestral y generalizada en todo el mundo, en especial en rituales mágicos y religiosos (Firenzouli et al. 2005) o como medicamentos (Li y Ohizumi 2004). El empleo de productos naturales empíricos fue y sigue siendo, una de las formas habituales para buscar aliviar sus dolencias y en algunos casos, curar enfermedades. Conscientes de esto y como estrategia fundamental de investigación orientada a lograr un salto en la calidad de vida de los pueblos del Chocó biogeográfico, el Instituto de Investigaciones Ambientales del Pacífico viene investigando y defendiendo la riqueza del conocimiento médico tradicional de las comunidades negras e indígenas de la región, que encuentra una de sus múltiples manifestaciones en el tratamiento que médicos tradicionales hacen a pacientes que sufren enfermedades de alta ocurrencia, como es el caso de la gastritis y/o úlcera péptica.

Las úlceras pépticas y gastritis son frecuentes en la población colombiana; de acuerdo con estudios recientes, en Colombia el $6,2 \%$ de las personas entre 6 y 69 años ha padecido alguna vez úlcera de estómago o intestino (Encuesta Nacional de Salud 2007.

La gastritis es una inflamación de la capa interior del estómago, que puede ocurrir de manera repentina (aguda) o gradual (crónica). La gastritis crónica ocurre en dos de cada 10.000 personas, mientras que la aguda es más común y ocurre en ocho de cada 1000 personas. La gastritis puede ser ocasionada por irritación debido a uso excesivo de alcohol, vómitos crónicos, estrés o el uso de ciertas medicinas como la aspirina y otras drogas antiinflamatorias, o por la presencia de Helicobacter pylori en el tracto gastrointestinal (Wallace y Granger 1996, Maity et al. 2003). Los síntomas de gastritis varían de individuo a individuo, y en mucha gente no hay síntomas, pero los más comunes incluyen: náusea, vómitos, indigestión, hipo, pérdida de apetito, sensación de inflación del abdomen, vómitos de apariencia sanguínea o de material de tipo café molido, deposiciones negras de consistencia oleosa.

Entre los metabolitos secundarios de plantas con actividad antigástrica tenemos: flavonoides (quercetina, narigina, similarina, antocianósidos y derivados de la sorofadina); saponinas, taninos, gomas y musilagos, alcaloides (matrina y oximatrina), aceites, triterpenoides (ácido oleanólico, acetato de $\beta$-lupeol, tarexerol y ácido ursólico).

En la medicina tradicional se ha reportado la acción antiinflamatoria y antigástrica de varios productos vegetales, que son usados por la población general como recursos terapéuticos por su fácil obtención y costo económico, refiriéndose mínimas reacciones adversas (Arellano 1993, Barriga 1985). Se ha estudiado el plátano (Musa sapientum $L$. musaceae) en varios modelos experimentales (Sanyal, Das, Sinha y Sinha 1961; Sanyal, Burnerjee y Das 1965), así como la col (Brassica oleracea L. cruciferae), que usada como antiulceroso llevó al desarrollo del gafarnate (Adami, MarzziUberti y Turba 1964). Sin embargo, en nuestro medio, existen pocos trabajos experimentales que corroboren los supuestos beneficios de diversas plantas, incluyendo caléndula (Calendula oficinales), llantén (Plantago major), botoncillo (Spilanthes americana) y micay (Axonopus micay).

En esta memoria se presentan los resultados obtenidos, en relación con la determinación de la efectividad de un tratamiento médico tradicional para la cura y/o alivio de la gastritis, utilizando una composición etnofarmacológica preparada a partir de cuatro especies de plantas medicinales (caléndula (Calendula oficinales), llantén(Plantago major), botoncillo (Spilanthes americana) y micay (Axonopus micay).

\section{OBJETIVO}

Evaluar la efectividad de una composición etnofarmacológica preparada a partir de cuatro especies de plantas medicinales: caléndula (Calendula oficinales), llantén (Plantago major), botoncillo (Spilanthes americana) y micay (Axonopus micay) para el tratamiento de la gastritis.

\section{METODOLOGÍA}

En este informe se presentan los resultados de esta investigación obtenidos en los municipios de Quibdó y Bagadó. El primero se encuentra ubicado en la región natural de la costa pacífica a $43 \mathrm{msnm}$ y sobre la margen derecha del río Atrato, a $5^{\circ} 40^{\prime} \mathrm{N}$ y $76^{\circ} 40^{\prime} \mathrm{O}$, su extensión total es de 6164 $\mathrm{km}^{2}$, presenta una humedad relativa de $86 \%$, una temperatura de $28^{\circ} \mathrm{C}$ y una precipitación de $10.749 \mathrm{~mm}$ anuales, presenta un número aproximado de 112.886 habitantes, según el censo del DANE (2005). Quibdó, por su extensión, comprende 


\section{Bioetnia Volumen 8 No 1 (enero-junio), 2011}

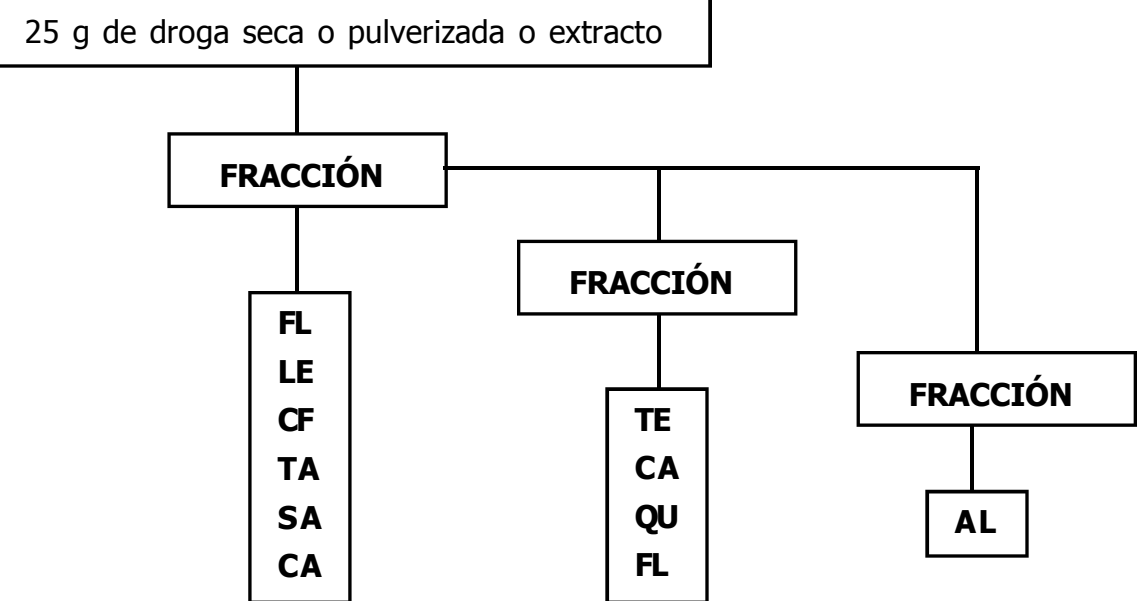

Figura 1. Flujograma resumen de la metodología utilizada para el análisis fitoquímico.

diversas clases de regiones donde predomina el bosque pluvial premontano (bp-PM), el bosque pluvial tropical (bpT) yel bosque muy húmedo montano bajo (bmh-MB) (Espinal 1997). El municipio de Bagadó se localiza en la margen izquierda del río Andágueda, a 5 $24^{\prime} 32^{\prime \prime} \mathrm{N}$ y $76^{\circ} 25^{\prime} 16^{\prime \prime} \mathrm{O}$. La altura sobre el nivel del mar es de $200 \mathrm{msnm}$. La temperatura media es de $28^{\circ} \mathrm{C}$. La precipitación media anual es de $7800 \mathrm{~mm}$ y dista de Quibdó 80 km (Arango y Saldarriaga 1997). Elárea municipal es de $777 \mathrm{~km}^{2}$, presenta una población 2372 habitantes, limita por el norte con Lloró y el Carmen de Atrato, por el este con el departamento de Antioquia, por el sur con Tadó y el departamento de Risaralda, y por el oeste con Tadó y Lloró (Arango y Saldarriaga 1997).

El desarrollo del estudio comprendió varias fases interrelacionadas; se inició con la identificación y descripción de las plantas medicinales utilizadas en la preparación de la composición etnofarmacológica usada para tratar la gastritis y se determinó el contenido químico de la misma, así como su efectividad.

Identificación y descripción de las plantas medicinales. Para este aspecto en particular y entendiendo el proceso progresivo en el que se desarrolló este proyecto, se procedió, en primer lugar, a tomar el registro de las plantas utilizadas en la preparación de la composición etnofarmacológica para el tratamiento de la gastritis, a fin de verificar su identificación taxonómica, completar las descripciones botánicas y hacer una revisión bibliográfica exhaustiva de la especie o en su defecto el género y/o la familia. En este sentido el material botánico se identificó en el herbario de la Universidad Tecnológica del Chocó, en algunos casos partiendo de su nombre vulgar y haciendo la respectiva verificación de la muestra y en otros haciendo comparación directa de las muestras con el material herbolario concentrado en esta colección botánica del Chocó biogeográfico.
Contenido químico de la composición etnofarmacológica. Antes de llevar a cabo el tamizaje fitoquímico a la composición etnofarmacológica, se realizó una revisión de literatura en algunas bases de datos (Current Contents 19982010), a fin de identificar estudios de análisis del contenido químico (metabolitos secundarios) y actividades biológicas de las plantas que conforman la composición etnofarmacológica para el tratamiento de la gastritis. Al igual que la realización de un ejercicio de vigilancia tecnológica aplicado a las propiedades de estas especies de plantas medicinales y a la efectividad de las terapias a partir de compuestos derivados de sus componentes, siguiendo de manera particular los lineamientos propuestos por la OMS en esta materia.

El material vegetal presente en la composición etnofarmacológica se sometió a análisis químico mediante marcha fitoquímica, utilizando los siguientes test de reconocimiento: reacción de cloruro férrico para compuestos fenólicos $(\mathrm{CF})$, reacción de proteínas para taninos (TA), reacción de Shinoda para flavonoides (FL), reacción de Rosenheim para leucoantocianidinas (LE), reacción de Kedde para compuestos lactónicos (CA), método de la espuma para saponinas (SA), reacción de Lieberman-Burchard para triterpenóides y/ o esteroides (TE), reacción de Borntranger para quinonas (QU), reacciones de Mayer, Valser, Reineckato de amonio y Dragendorff para alcaloides (AL). Todos estos análisis se realizaron por triplicado (Figura 1).

Determinación de la efectividad del tratamiento médico tradicional aplicado a la cura y/o alivio de la gastritis. Para avanzar en la determinación de la efectividad del tratamiento médico tradicional usado para tratar la gastritis, se diseñaron y aplicaron protocolos de entrevistas individuales a pacientes declarados portadores de estas dolencias por parte de un médico facultativo y sometidos al tratamiento médico tradicional propuesto, que consistió en el suministro de una dosis 
Tabla 1

Plantas medicinales utilizadas en la composición etnofarmacológica para el tratamiento de la gastritis

\begin{tabular}{cccc}
\hline No & Nombres comunes & Nombre científico & Parte utilizada \\
\hline 1 & $\begin{array}{l}\text { Caléndula } \\
\text { Llantén, plantago, arta, } \\
\text { plantaina, siete vena }\end{array}$ & Calendula officinales & Flores \\
\hline 3 & $\begin{array}{l}\text { Botoncillo, chisacá, guaca, } \\
\text { risaca, quemadera, tuyo, } \\
\text { orosus, }\end{array}$ & Hojas \\
\hline 4 & Micay & Spilanthes americana & Hojas \\
\hline
\end{tabular}

diaria (un vaso aproximadamente o $250 \mathrm{ml}$ ) de la composición etnofarmacológica en estudio, que se debía tomar especialmente antes de acostarse, con recomendaciones como las de no consumir granos, ni tomar bebidas alcohólicas. A estos pacientes se les realizó seguimiento mediante expediente clínico, a fin de sistematizar y documentar los procedimientos médicos tradicionales, en términos de sus componentes, eficacia y dosificaciones.

\section{RESULTADOSYDISCUSIÓN}

Identificación y descripción de las plantas medicinales. La composición etnofarmacológica, está compuesta por una combinación de cuatro extractos acuosos de plantas medicinales (Tabla 1): extracto acuoso de flores de caléndula (Calendula oficinales), hojas de llantén (Plantago major), hojas de botoncillo (Spilanthes americana) y planta completa de micay (Axonopus micay).

Caléndula(Calendula oficinales), de la familia Asteraceae, es una planta herbácea anual, que alcanza una altura de $30 \mathrm{~cm}$ a $60 \mathrm{~cm}$. Posee tallos robustos, vellosos, angulosos, en los que se insertan las hojas enteras o ligeramente dentadas y oblongas, espatuladas las de la base. Los brotes terminan en capítulos solitarios, de unos cuatro centímetros de diámetro, formado de flores de color amarillo a anaranjado. Los frutos son aquenios y espinosos.

Llantén(Plantago major), de la familia Plantaginaceae, es una hierba rosetada desde un grueso rizoma; hojas simples, numerosas, ampliamente ovadas y redondeadas en la base, tosca e irregularmente dentada en el margen, con pedúnculos hasta de $15 \mathrm{~cm}$ de largo, flores amarillentas escariosas agrupadas en espigas.

Botoncillo (Spilanthes americana), de la familia Asteraceae, es una hierba de $40 \mathrm{~cm}$ de altura, hojas opuestas, pecioladas, oblongas, agudas, aserradas, base redonda, flo- res amarillas de discos abundantes.

Micay (Axonopus micay), de la familia Gramineaceae, es una planta perenne de porte bajo, aislada, con tallos postrados; generalmente los tallos no producen raíces en los nudos, la espiga es semejante a la del pasto imperial, aunque tiene un número mayor de espiguillas.

Contenido químico de la composición etnofarmacológica. La revisión de literatura (Current Contents 19982010) referida a estudios de análisis del contenido químico (metabolitos secundarios) y actividades biológicas de las plantas que conforman la composición etnofarmacológica para el tratamiento médico tradicional usado para la cura y/o alivio de la gastritis, nos muestra que algunas de estas plantas contienen en su estructura química metabolitos secundarios tales como: flavonoides, derivados triterpénicos, leucoantocianidinas, saponinas, compuestos fenólicos, antocianinas, entre otros (Tabla 2).

Los análisis fitoquímicos realizados a la composición etnofarmacológica, mostraron resultados de presunción positiva para la presencia de compuestos fenólicos, cumarínas y leucoantocianidinas. Además, es probable la presencia de triterpenoides o esteroides aunque la prueba no arroja resultados contundentes (Tabla 3).

El resultado de estos análisis fitoquímicos (Tabla 3), junto con información bibliográfica reciente sobre estudios fitoquímicos y farmacológicos de las especies vegetales usadas en la preparación de esta composición etnofarmacológica (Tabla 2), demuestran la presencia de saponinas y glicósidos triterpenoides, sobre todo en las flores de caléndula (Calendula officinalis) (Yoshikawa, Murakami y Kishi et al. 2001), extracto metanólico en hojas de llantén (Plantago major) (Yesilada etal. 1993).

Determinación de la efectividad del tratamiento médico tradicional aplicado a la cura y/o alivio de la gastritis. Para avanzar en la determinación de la efectividad del tratamiento 
Bioetnia Volumen 8 No 1 (enero-junio), 2011




Composición etnofarmacológica chocoana, alternativa para aliviar la gastritis. CA. Rentería, MA. García

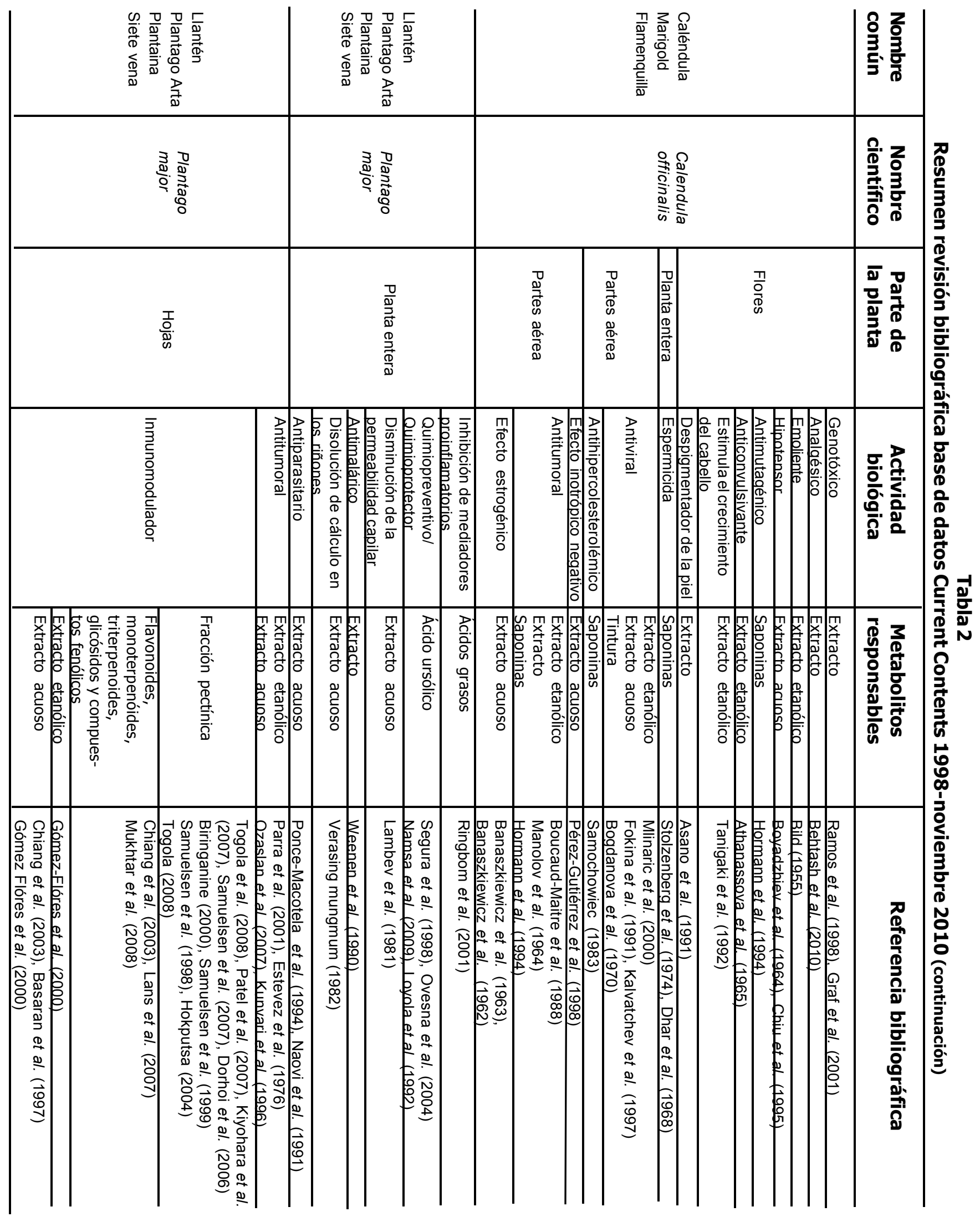


Bioetnia Volumen 8 No 1 (enero-junio), 2011

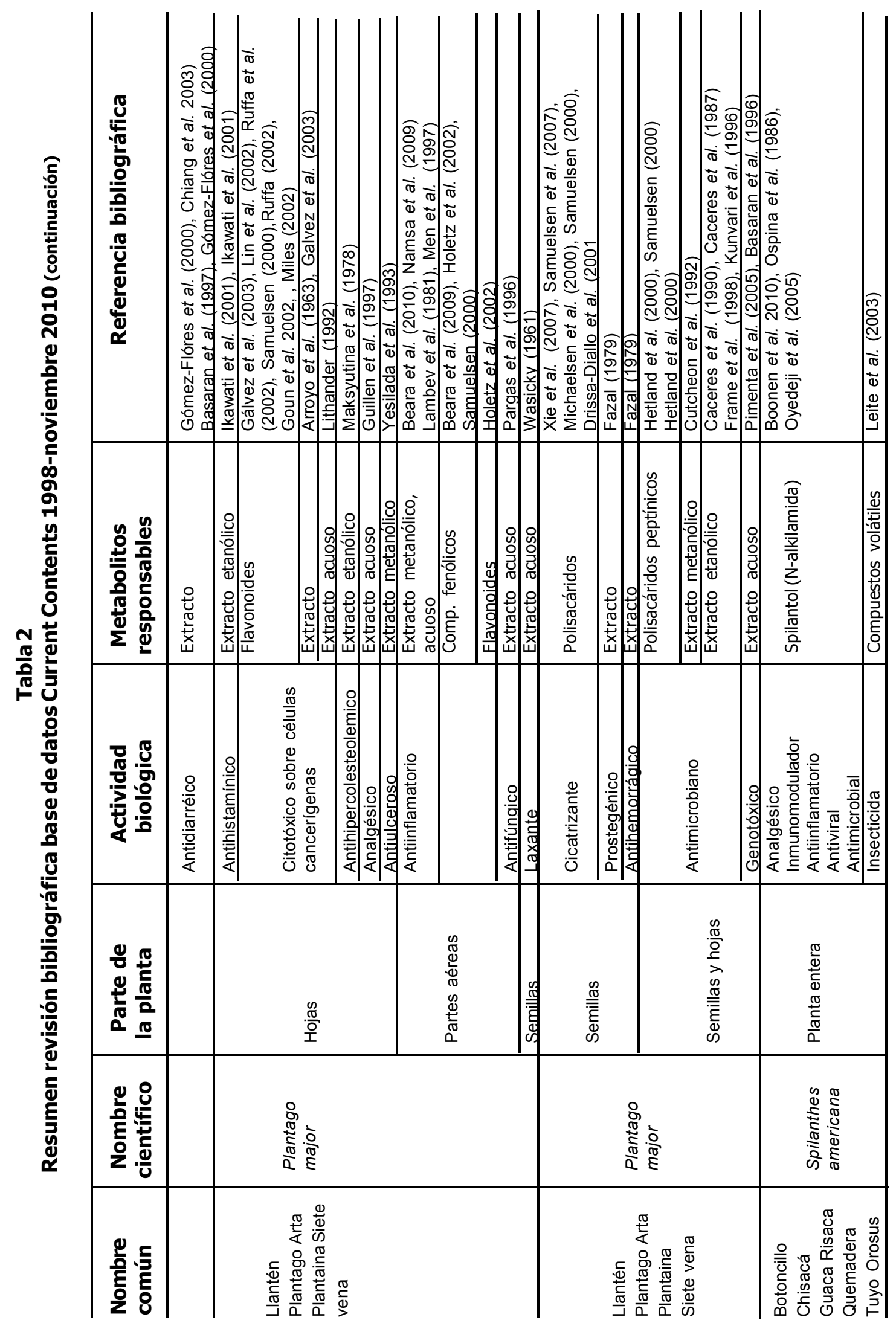


Tabla 3

Resultados marcha analítica de la composición etnofarmacológica para el tratamiento de la gastritis

Prueba

\begin{abstract}
Extracto acuoso
(Fracción A)
\end{abstract}

Solución orgánica

(Fracción B)
Solución acuosa ácida

(Fracción C)

$\begin{array}{lcc}\text { Compuestos fenólicos } & ++ & \\ \text { Cumarínas } & + & \\ \text { Leucoantocianidinas } & ++ & \\ \text { Saponinas } & - & - \\ \text { Taninos } & - & - \\ \text { Flavonoides } & - & +/- \\ \text { Compuestos cardiotónicos } & & - \\ \text { Triterpénos y/o esteroides } & & \\ \text { Quinonas } & & - \\ \text { Alcaloides } & \end{array}$

Negativo (-), Positivo (+), Muy positive (++), Altamente positive (+++), Dudoso (+/-)

médico tradicional usado para tratar la gastritis, se realizó un monitoreo acompañado de entrevistas a catorce pacientes, todos declarados con síntomas de gastritis por parte de un médico facultativo, pacientes que participaron de manera informada y voluntaria en la investigación. Estas catorce personas tomaron durante cerca de 20 días, antes de acostarse, $200 \mathrm{ml}$ a $250 \mathrm{ml}$ (un vaso aproximadamente) de la composición etnofarmacológica, se describieron y anotaron los síntomas descritos por los pacientes antes de iniciar el tratamiento y luego a los 5, 10, 15 y 20 días de continuado el mismo, se registraron resultados progresivamente a través de entrevistas.

En las diferentes pruebas (entrevistas a los pacientes) realizadas para evaluar su efectividad y eficacia, la composición etnofarmacológica mostró tener propiedades para aliviar los síntomas producidos por la gastritis, además de un amplio espectro antigástrico. Se observó que la composición tuvo una efectividad bastante significativa en relación con el tratamiento y las personas mostraron porcentajes de recuperación y mejoría sintomática relacionados con la gastritis, lo que se evidencia sobre todo en los testimonios de los pacientes (Tabla 4).

La composición etnofarmacéutica mostró tener actividad clínica para aliviar y tratar la gastritis sin causar efectos indeseables en el individuo al que se le suministra.

\section{CONCLUSIONES}

Esta investigación permitió conocer la función terapéutica de algunas plantas medicinales, para aliviar y/o curar la gastritis. Después de analizar los diferentes testimonios de las personas que se sometieron al tratamiento se pudo corroborar que, efectivamente, funciona muy bien cuando este es aplicado de manera correcta y con las medidas indicadas para su uso, mostrando así una mejoría en las personas que padecían estas enfermedades.

Información bibliográfica reciente sobre estudios fitoquímicos y farmacológicos de las especies vegetales usadas en la preparación de estas composiciones etnofarmacológicas, demuestran la presencia de saponinas, esteroles y flavonoides, principalmente en las hojas de caléndula, llantén y botoncillo, compuestos con reconocida actividad antigástrica, lo que se confirmó por los testimonios de muchas de las personas que se han sometido al tratamiento etnofamacológico descrito en este estudio.

En diferentes pruebas realizadas a pacientes con gastritis, la composición etnofarmacéutica mostró tener propiedades para aliviar algunos de los síntomas producidos por esta enfermedad y además, un amplio espectro antigástrico, pues se observó que alivió síntomas propios de la gastritis, como sensación de ardor y nauseas. La efectividad que se obtuvo fue bastante significativa y las personas mostraron porcentajes de recuperación y disminución de los síntomas, como se puede concluir de los testimonios de los pacientes sometidos a este tratamiento. Sin embargo, es importante realizar estudios farmacológicos y clínicos que complementen esta información.

\section{AGRADECIMIENTOS}

Los autores agradecen al Grupo de Investigación de Productos Naturales Marinos de la Universidad de Antioquia y en especial al Elkin Galeano, MSc por su valiosa colaboración. 
Bioetnia Volumen 8 No 1 (enero-junio), 2011

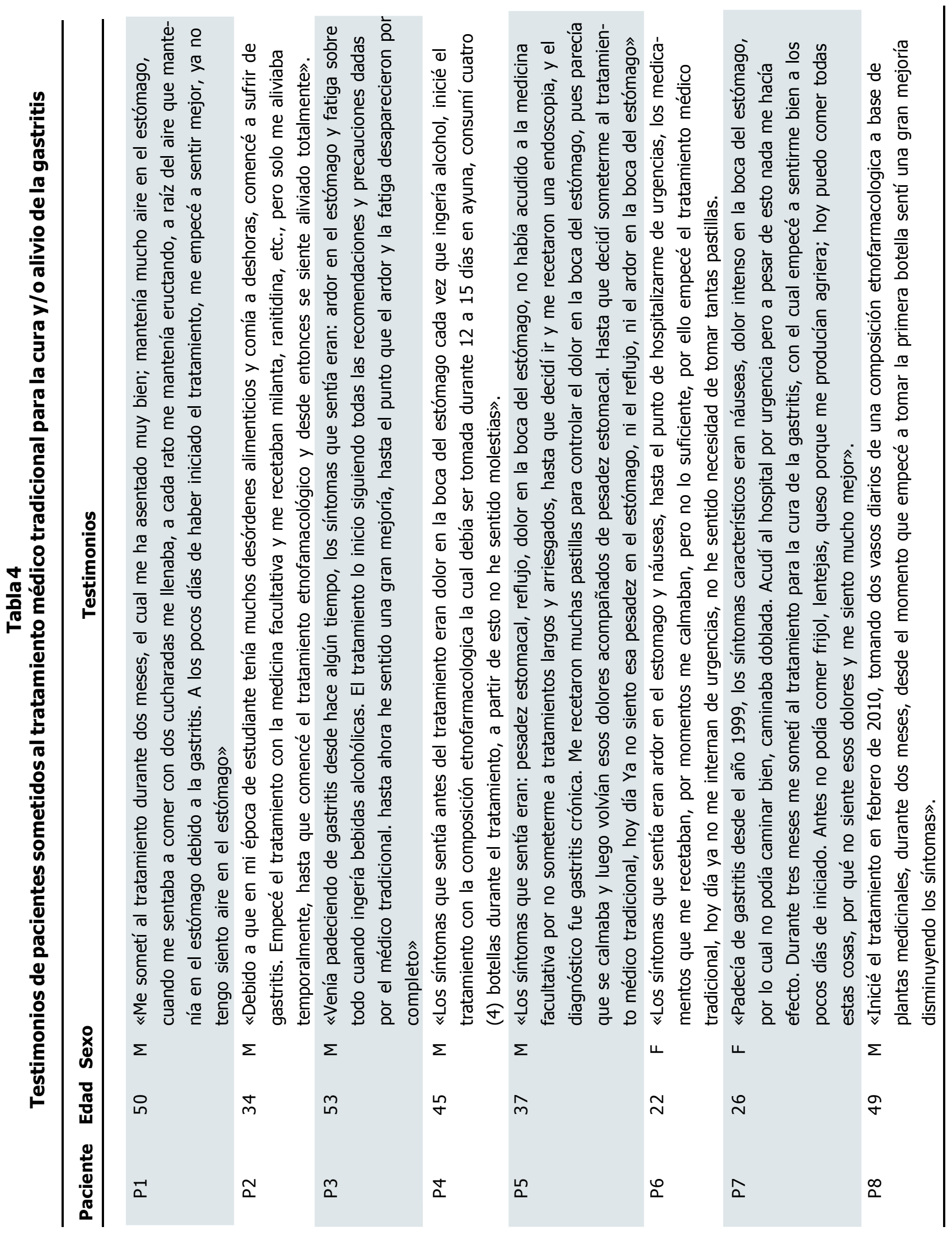




\section{Bioetnia Volumen 8 № 1 (enero-junio), 2011}

\section{LITERATURA CITADA}

Adami, E., E. Marzzi-Uberti, C. Turba. 1964. Pharmacological research on gafarnate, a new synthetic isopenoid with an antiulcer action. Arch Intern Pharmacodynamie Ther. 147: 113-45.

Arango, S, Saldarriaga, S.T. 1997. Monografia del Chocó. Quibdó: Editorial de Autores Chocoanos.

Arellano, P. 1992. El libro verde. Guía de recursos terapéuticos vegetales. Lima: Ministerio de Salud. p. 33, 46, 53.

Barriga, R. 1994. Plantas útiles de la Amazonía peruana, características, usos y posibilidades. Trujillo: CONCYTEC. $261 \mathrm{pp}$.

Firenzuoli, F., I. Gori, D. Neri. 2005. Clinical phytotherapy: opportunities and problematics. Ann Ist Super Sanita. 41 (1): 27-33.

Instituto de Investigaciones Ambientales del Pacífico. 2010. Validación de tratamientos basados en medicina tradicional, dirigidos a la cura de enfermedades de alta ocurrencia nacional y mundial. Hacia la obtención de patentes etnofarmacológicas. Informe Final. Chocó: IIAP.

Instituto de Investigaciones Ambientales del Pacífico. Universidad de Antioquia. 2010. Informe final Convenio Interadministrativo de Cooperación e Investigación. Chocó: IIAP.

Li, Y., Y. Ohizumi. 2004. Search for constituents with neutrophic factor potentiating activity from the medicinal plants of Paraguay and Thailand. Yakugaku Zasshi. 124 (7): 417-24.

Maity, P., K. Biswas, S. Roy, R. K. Banerjee, U. Bandyopadhyay. 2003. Smoking and the pathogenesis of gastroduodenal ulcer-recent mechanism update. Moll Cell Biochem. 253: 329-38.

Sanyal. A. K., C. R. Burnerjee, P. K. Das. 1965. Studies on peptic ulceration. Part II. Role of banana in restraint and prednisolone induced ulcer in albino rats. Arch Intern Pharmacodynamie Ther. 155: 244-8.

Wallace, J. L., D. N. Granger. 1996. The cellular and molecular basis of gastric mucosal defense. FASEB J. 10: 731-40. 